\title{
Isolated tricuspid valve surgery after congenital versus left heart- disease surgery: mid-term outcomes
}

\author{
Hong Cao ${ }^{1 \#}$, Xiaoxi Yang ${ }^{2 \#}$, Pablo Maureira ${ }^{3}$, Jinping Liu ${ }^{4}$, Junwei Liu ${ }^{1}$, Pan Dan ${ }^{3,4}$, Maxime Hubert ${ }^{3}$, \\ Jean-Pierre Villemot ${ }^{3}$, Nianguo Dong ${ }^{1}$, Yihua Liu ${ }^{1,3}$ \\ ${ }^{1}$ Department of Cardiovascular Surgery, Wuhan Union Hospital, Huazhong University of Science and Technology, Wuhan, China; ${ }^{2}$ Department \\ of Radiation and Medical Oncology, Zhongnan Hospital, Wuhan University, Wuhan, China; ${ }^{3}$ Department of Cardiovascular Surgery and Heart \\ Transplantation, University Hospital of Brabois, Vandoeuvre-les-Nancy, France; ${ }^{4}$ Department of Cardiovascular Surgery, Zhongnan Hospital, \\ Wuhan University, Wuhan, China \\ Contributions: (I) Conception and design: Y Liu, N Dong, P Maureira, JP Villemot; (II) Administrative support: N Dong; (III) Provision of study \\ materials or patients: J Liu, J Liu, N Dong; (IV) Collection and assembly of data: H Cao, Y Liu; (V) Data analysis and interpretation: X Yang, P Dan, \\ M Hubert; (VI) Manuscript writing: All authors; (VII) Final approval of manuscript: All authors. \\ \#These authors contributed equally to this work. \\ Correspondence to: Dr. Yihua Liu, Dr. Nianguo Dong. Department of Cardiovascular Surgery and Heart Transplantation, Union Hospital, Tongji \\ Medical College, Huazhong University of Science and Technology, 1277 Jiefang Road, Wuhan, China. \\ Email: arthur1232003@hotmail.com; dongnianguo@hotmail.com.
}

Background: Left heart involvement might be a differential factor in the physiopathology and prognosis of severe tricuspid regurgitation (TR) following cardiac surgery. We aimed to compare the outcomes of isolated tricuspid valve surgery (ITVS) after congenital versus left heart-disease surgery.

Methods: We retrospectively studied and followed up 58 patients who underwent ITVS for TR following cardiac surgery in our center from January 2012 to December 2017. According to the different etiologies of TR, the participants were divided into one group of TR following surgery for congenital heart diseases (CHD) (pCHD group, $n=24$ ), and another group of TR following surgery for left heart disease (pVHD group, $\mathrm{n}=34)$.

Results: Compared to the pCHD group, the pVHD group presented with a more advanced age $(\mathrm{P}<0.001)$, higher model for end-stage liver disease (MELD) score calculation ( $\mathrm{P}=0.04)$, and higher EuroSCORE II calculation $(\mathrm{P}=0.01)$. In the post-operative course, the $\mathrm{pVHD}$ group showed a longer mechanical ventilation time $(\mathrm{P}<0.001)$ and longer intensive care unit stay $(\mathrm{P}=0.001)$. However, there was no significant difference between the two groups in in-hospital mortality $(8.8 \%$ vs. $0, \mathrm{P}=0.26)$, or the incidence of major adverse cardiac and cerebrovascular events (MACCE) (20.6\% vs. $12.5 \%, \mathrm{P}=0.47)$ at follow-up.

Conclusions: Severe TR following surgery for left heart disease is associated with higher surgical risks and a remarkable frailty as compared to that following surgery for CHDs; however, with the development of surgical techniques and peri-operative management, ITVS can be safely performed in both conditions with promising contemporary mid-term outcomes.

Keywords: Tricuspid regurgitation (TR); valvular heart diseases; congenital heart diseases (CHD); redo surgery

Submitted Apr 28, 2020. Accepted for publication Sep 02, 2020.

doi: $10.21037 /$ jtd-20-1787

View this article at: http://dx.doi.org/10.21037/jtd-20-1787

(c) Journal of Thoracic Disease. All rights reserved. 


\section{Introduction}

Severe tricuspid regurgitation (TR) may result in progressive and retractable right heart failure, and is thus associated with poor survival regardless of left ventricular function and pulmonary hypertension (1). According to the anatomical abnormality of tricuspid valve apparatus, TR is typically divided into primary and secondary TR. Recent guideline recommendations favor an aggressive surgical intervention in patients with severe symptomatic TR $(2,3)$. Concomitant tricuspid valve surgery to manage secondary TR has gained remarkable popularity in the modern era; however, isolated tricuspid valve surgery (ITVS) remains an infrequent procedure as a result of perceived high surgical mortality. The surgical risks related to ITVS are predominantly entwined with those of redo surgery, visceral dysfunction secondary to venous congestion, and the general frailty of recipients. Theoretically, TR following left-sided valvular (mitral and/or aortic valve) surgery compared to that following congenital heart disease (CHD) surgery is associated with even more risks due to left heart structural and/or functional impairments and pulmonary circulation involvement. Our study aimed to evaluate the mid-term all-cause mortality and incidence of major adverse cardiac and cerebrovascular events (MACCEs) following ITVS for severe TR that ensued from cardiac surgery for different etiologies. We present the following article in accordance with the Strengthening The Reporting of Observational Studies in Epidemiology (STROBE) reporting checklist (available at http://dx.doi.org/10.21037/jtd-20-1787).

\section{Methods}

\section{Study design}

We conducted a retrospective observational study of patients referred to our department for surgical management of severe TR between January 2012 and December 2017. The study was conducted in accordance with the tenets of the Declaration of Helsinki (as revised in 2013), and was approved by the institutional ethics committee (No: IORG0003571). Informed consent was waived because of the retrospective nature of the study and the use of anonymous clinical data for analysis.

\section{Patients}

Participant inclusion criteria were the following: (I) older than 18 years of age; (II) previous cardiac surgery of any cause; (III) undergone ITVS for severe TR following cardiac surgery. The exclusion criteria were the following: (I) concomitant cardiac surgery such as coronary artery bypass grafting, left-sided valve repair, or correction of congenital malformation; (II) native tricuspid valve stenosis. According to the etiologies, the patients were divided into two groups: (I) post-operative TR following surgery for CHDs (pCHD, $\mathrm{n}=24$ ) and (II) post-operative TR following surgery for leftsided valvular heart diseases (pVHD, n=34) (Figure 1). In the pre-operative period, all patients received optimized medical management including intravenous infusion of diuretics and dobutamine to reduce volume overload of the right heart and to improve the functional status.

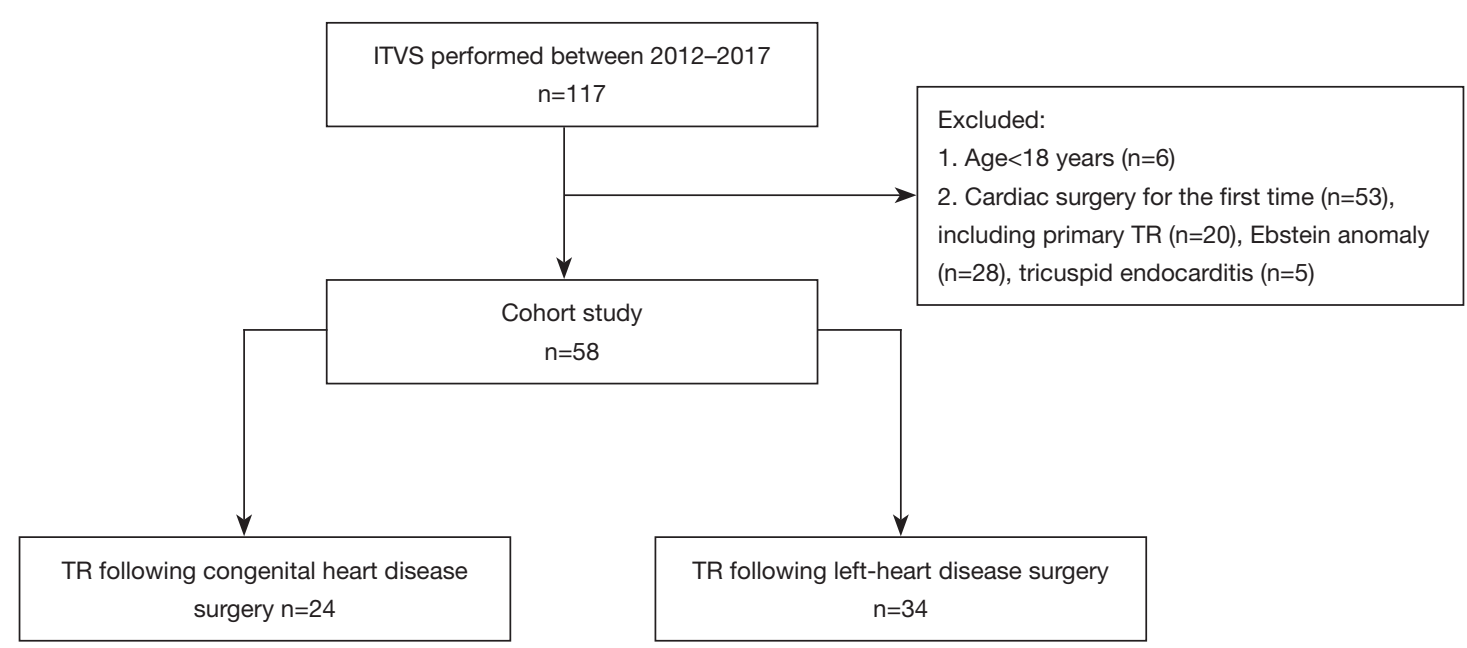

Figure 1 Flow diagram illustrating patients 'inclusion and exclusion. ITVS, isolated tricuspid valve surgery; TR, tricuspid regurgitation. 


\section{Variables}

Preoperative demographics and clinical characteristics, echocardiographic data, and operative parameters were collected for analysis. The model of end-stage liver disease (MELD) score was used to evaluate the visceral dysfunction, and the EuroSCORE II calculation was used as a predictor of surgical risks. The echocardiographic variables included the end-diastolic diameter of four heart chambers, left ventricular ejection fraction, the estimation of systolic pulmonary artery pressure derived from the continuous wave Doppler velocity of the tricuspid regurgitant jet, and right ventricular function assessed by eyeballing and gross categorization as normal, mild, moderate, or severe impairment. The surgical strategies for the type of surgery (tricuspid repair or replacement), surgical approach (sternotomy or thoracotomy), use of aortic cross-clamping, and the choice of implanted materials (band, ring, tissue prosthesis, or mechanical prosthesis) were included in the operative variables.

\section{Follow-up}

Follow-up was completed by routine out-patient visits and telephone interviews. All patients underwent at least one echocardiographic evaluation at 3-month post-operative intervals. The primary endpoint was all-cause mortality during follow-up. The secondary endpoint was a composite of all MACCEs including repeated hospitalization for heart failure, reoperation for prosthetic dysfunction or failed repair, cardiac death, and stroke.

\section{Statistical analysis}

Continuous normally distributed variables are expressed as mean \pm standard deviation, while non-normally distributed variables are presented as median (first quartile-third quartile). The assumption of normality was assessed using the Shapiro-Wilk test. Categorical variables are presented as numbers (\%). The missing values were excluded from further analysis. Continuous normally and nonnormally distributed variables were compared using the Student's $t$-test and non-parametric Wilcoxon rank-sum test, respectively. Categorical variables were compared using Pearson's Chi-squared or Fisher's exact test where appropriate. The actuarial estimate of survival was made using the Kaplan-Meier method, and Kaplan-Meier survival curves were compared with the log-rank test. Univariate and multivariable analyses were performed using the Cox proportional hazards model to identify predictors of the primary and secondary endpoints. A two-tailed $\mathrm{P}$ value $<0.05$ was considered statistically significant. Statistical analysis was performed using the statistical Package for Social Science, version 23.0 (SPSS, Chicago, IL, USA).

\section{Results}

\section{Demographics and clinical characteristics}

The mean age of the participants was $46.5 \pm 13.7$ years, and $36.2 \%(21 / 58)$ were male patients. Of the participants, $58.6 \%(34 / 58)$ were in the New York Heart Association (NYHA) class III/IV and the mean EuroSCORE II calculation was $2.7 \% \pm 0.8 \%$. Patients typically had mild pulmonary hypertension with a mean systolic pulmonary artery pressure of $40.2 \pm 12.1 \mathrm{mmHg} ; 13.8 \%(8 / 58)$ patients presented with moderate/severe right ventricular dysfunction as evaluated by echocardiography, and $37.9 \%$ (22/58) presented with chronic atrial fibrillation. Other baseline characteristics are shown in Table 1. Significant difference was observed between the two groups in age, incidence of atrial fibrillation, MELD score, EuroSCORE II calculation, and left atrial diameter. In general, the pCHD group presented with better functional status and less predictive surgical risks compared to the pVHD group.

\section{Surgical indications and technical variables}

As shown in Table 2, in the pCHD group, 4, 7, and 2 patients had undergone previous surgical correction for atrial septal defects, ventricular septal defects, and atrioventricular canal defects, respectively; 11 patients had been operated on for Ebstein anomaly, 3 of whom suffered from dysfunction of mechanical tricuspid prostheses as a result of pannus formation; and 8 patients developed progressive TR following tricuspid repair. In the pVHD group, 18 and 16 patients had undergone mitral and mitroaortic valve replacement, respectively. IVTS was performed via median sternotomy in nearly $80 \%$ of patients; however, in the last 3 years, right thoracotomy had increased in popularity. Tricuspid valve replacement was the preferred surgical strategy accounting for almost $90 \%(52 / 58)$ of the entire cohort, and tissue prostheses $(53.4 \%)$ were more frequently employed than mechanical (36.2\%). IVTS was performed on $42(72.4 \%)$ patients without the application of ascending aorta cross-clamping. 
Table 1 Demographics, clinical characteristics, and echocardiographic data

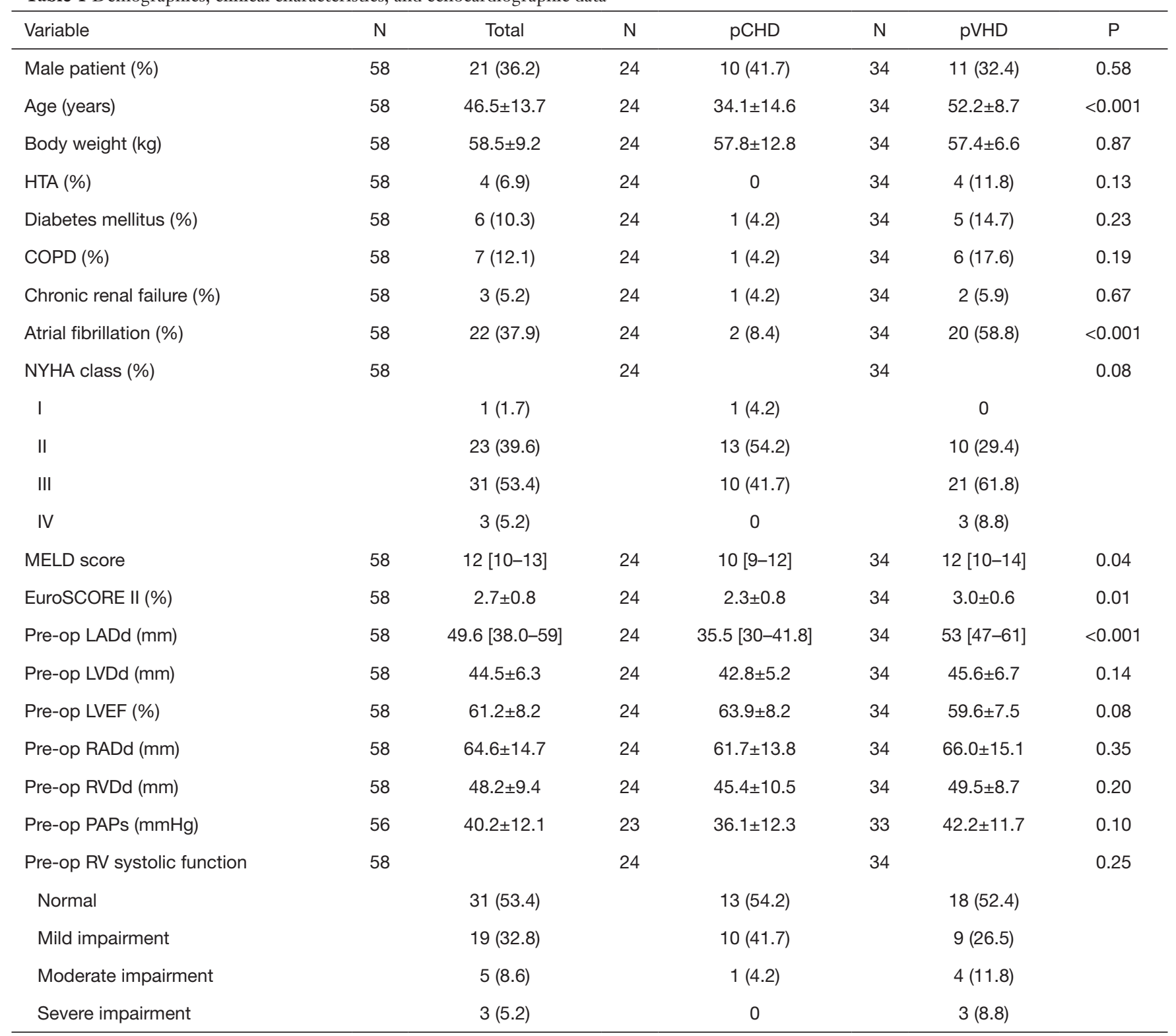

CHD, congenital heart disease; HTA, arterial hypertension; COPD, chronic obstructive pulmonary diseases under bronchodilator medications; NYHA, New York Heart Association; MELD score, model for end-stage liver disease score; pre-op, pre-operative; LADd, diastolic dimension of left atrium; LVDd, diastolic dimension of left ventricle; LVEF, left ventricular ejection fraction; RADd, diastolic dimension of right atrium; RVDd, diastolic dimension of right ventricle; PAPs, systolic pulmonary arterial pressure.

\section{Postoperative complications and in-hospital outcomes}

High grade atrio-ventricular block requiring definitive pacemaker implantation $(8.6 \%, 5 / 58)$ was the most frequent post-operative complication, followed by continuous renal replacement therapy for acute renal failure $(5.2 \%, 3 / 58)$, mediastinal bleeding $(3.4 \%, 2 / 58)$, and sternal wound infection $(3.4 \%, 2 / 58)$. The incidences of major post- operative complications were comparable in the two groups (Table 3), but the intensive care unit stay and postoperative in-hospital stay were significantly longer in the pVHD group. In our series, the echocardiography of control did not evidence either para-prosthetic leakage or more-thanmoderate residual TR. The in-hospital mortality rate was $5.2 \%(3 / 58)$, with all deaths occurring in the pVHD group; 
Table 2 Indications and surgical parameters

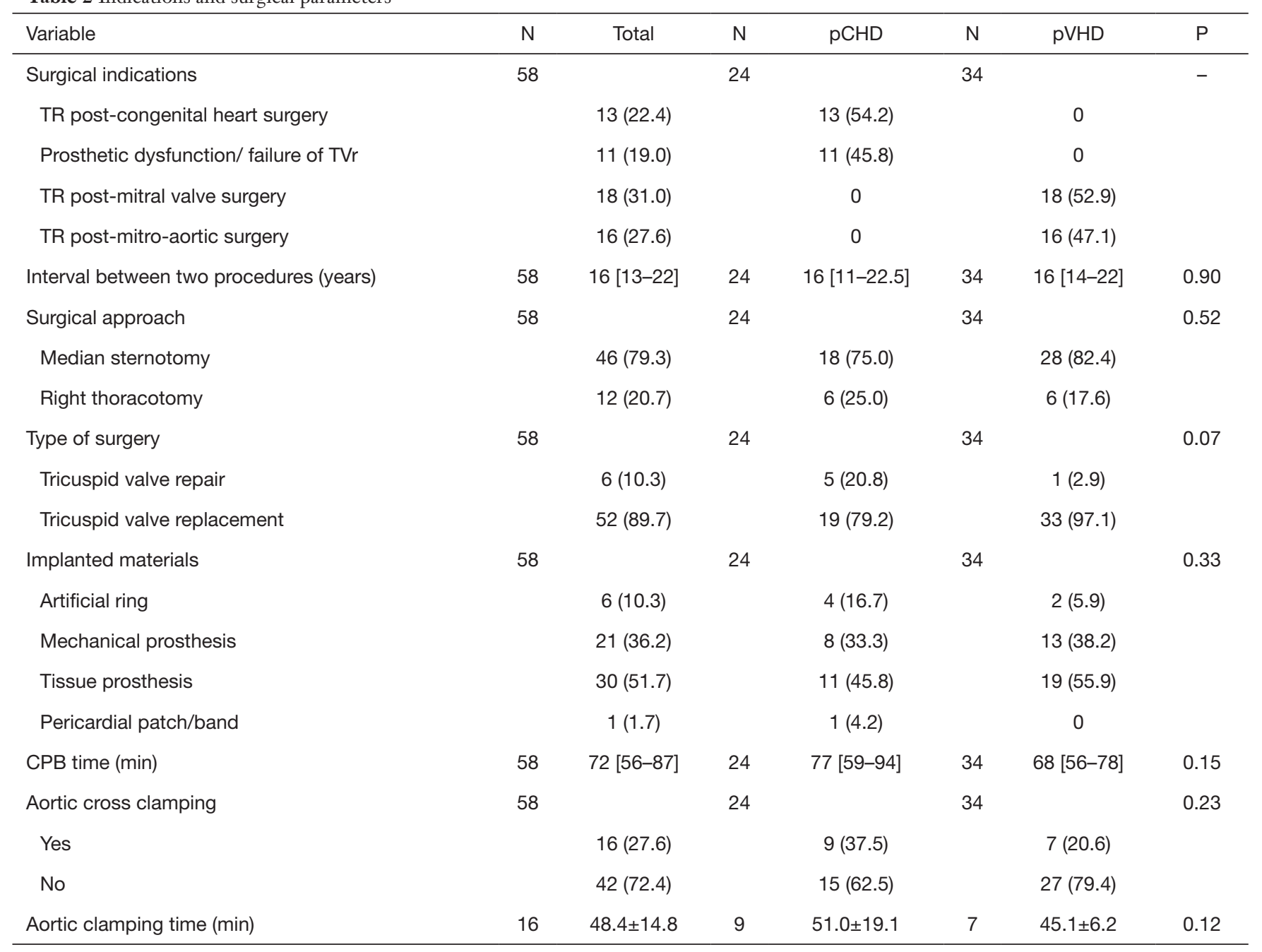

CHD, congenital heart disease; TR, tricuspid regurgitation; TVr, tricuspid valve repair; CPB, cardio-pulmonary bypass.

however, the difference was not statistically significant $(\mathrm{P}=0.26)$. All three mortalities resulted from low cardiac output syndrome with reserved left ventricular function, with the severely impaired right ventricular systolic function significantly reducing the pre-load of left hearts.

\section{Follow-up outcomes}

The follow-up rate was $96.5 \%(56 / 58)$, and the median follow-up duration was 47.8 months. During follow-up, 1 death was observed in the pVHD group at the $43^{\text {rd }}$ month post-operatively as a result of progressive heart failure. The Kaplan-Meier analysis showed a trend of higher all-cause mortality (Figure $2 A$ ) in the pVHD group, as compared to the pCHD group $(\mathrm{P}=0.09)$. During follow-up, cardiac death, re-operation for prosthetic dysfunction, stroke, and repeated hospitalization for heart failure were observed in 4, 2, 2, and 2 patients, respectively. The rates of MACCE during follow-up (Figure $2 B$ ) were not significantly different between the two groups $(\mathrm{P}=0.47)$. The univariate Cox regression analysis identified the risk factors affecting outcomes, including the interval between two cardiac interventions, MELD score, history of chronic obstructive pulmonary disease (COPD) and atrial fibrillation, EuroSCORE calculation, pre-operative NYHA class, preoperative left atrial diastolic dimension, pre-operative right ventricular dysfunction, duration of cardiopulmonary bypass time, mechanical ventilation time, and post-operative acute renal failure requiring continuous renal replacement therapy (Table 4). Nevertheless, due to the low incidence of 
Table 3 Post-operative clinical variables

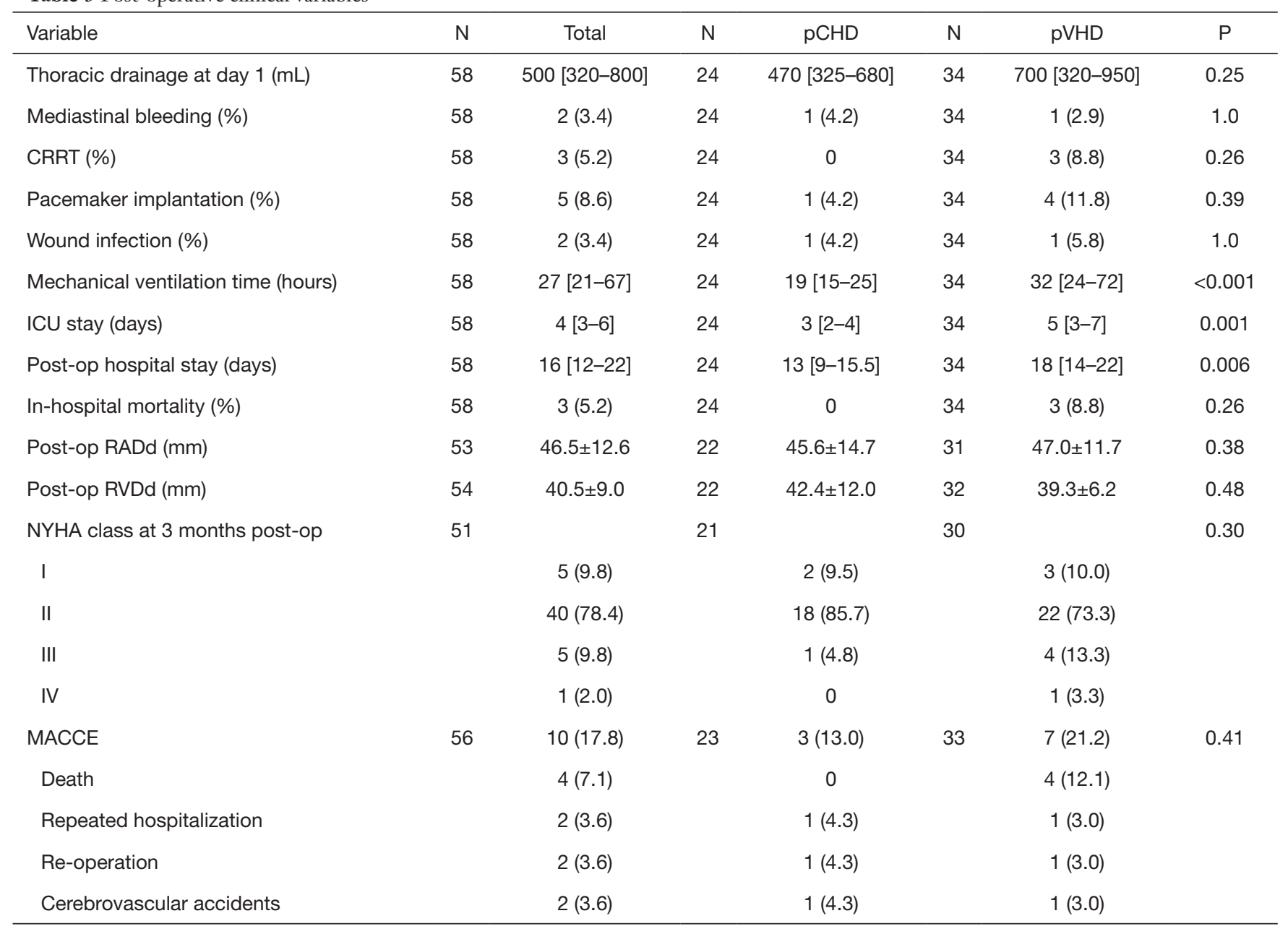

CHD, congenital heart disease; CRRT, continuous renal replacement therapy; ICU, intensive care unit; post-op, post-operative; RADd, diastolic dimension of right atrium; RVDd, diastolic dimension of right ventricle; MACCE, major adverse cardiac and cerebrovascular events.

the primary and secondary endpoints, we failed to identify any independent predictors for in-hospital mortality or MACCE incidence (Table 5).

\section{Discussion}

In this retrospective study, we compared the clinical profiles and mid-term outcomes of patients who underwent ITVS for severe TR following two types of intracardiac surgery: CHD surgery and left heart valvular surgery. We found that the patients who underwent prior left heart valvular surgery, as compared to those with previous cardiac surgery for CHDs, presented with higher surgical risks including advanced age, more comorbidities (MELD scores), and higher predictive mortality (EuroSCORE II calculation). However, with the evolution of surgical techniques and optimal peri-operative medical management, the mid-term outcomes, including overall mortality and MACCEs, were comparable between the two groups.

The last two decades have seen an increased understanding of the long-term adverse consequences of severe TR with remarkable advances in its surgical management and aggressive treatment recommendations. However, ITVS in contemporary practice is still associated with significant post-operative and long-term mortality. The risks relate not only to the surgery itself but also on the underlying complex pathophysiological changes, especially in the case of multiple organ involvement of the liver, 
A

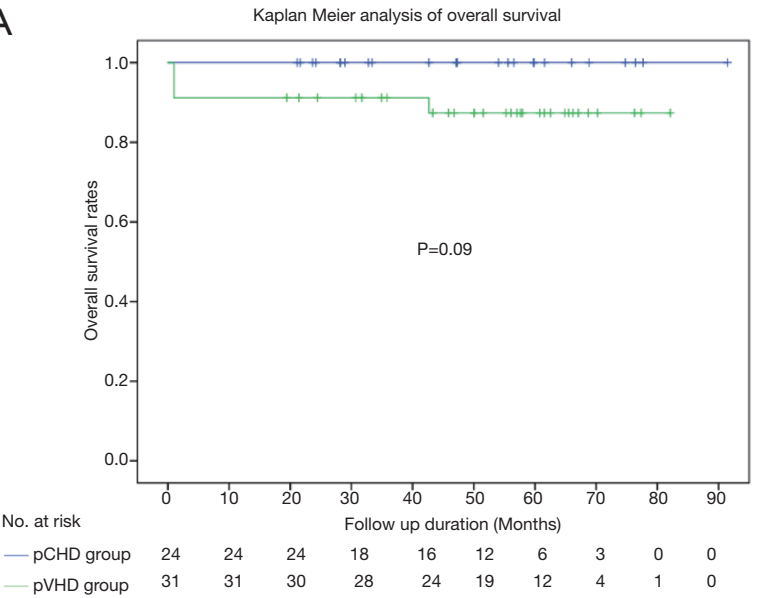

B

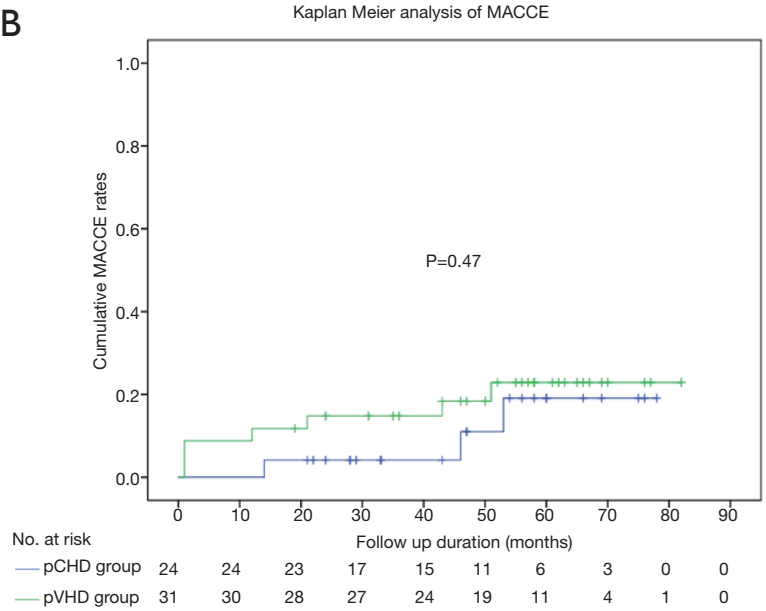

Figure 2 Kaplan-Meier analysis of overall survival (A) and MACCE incidence (B). MACCE, major adverse cardiac and cerebrovascular event.

Table 4 Univariate Cox regression for all-cause mortality and MACCEs

\begin{tabular}{|c|c|c|c|c|}
\hline Variables & \multicolumn{2}{|c|}{ For overall mortality } & \multicolumn{2}{|c|}{ For MACCEs } \\
\hline Interval between two procedures & $1.07(0.93-1.23)$ & 0.36 & $1.08(0.99-1.18)$ & 0.09 \\
\hline MELD score & $1.42(1.07-1.90)$ & 0.02 & $1.23(1.02-1.48)$ & 0.03 \\
\hline COPD & $7.89(1.10-56.60)$ & 0.04 & $4.09(1.05-15.98)$ & 0.04 \\
\hline EuroSCORE II & $4.0(1.16-13.84)$ & 0.03 & $1.83(0.76-4.42)$ & 0.18 \\
\hline Pre-op NYHA class & $14.06(2.25-87.92)$ & 0.005 & $5.15(1.53-17.31)$ & 0.008 \\
\hline Pre-op LADd & $1.07(1.02-1.13)$ & 0.01 & $1.04(1.00-1.07)$ & 0.046 \\
\hline Right ventricular dysfunction & $3.73(1.45-9.59)$ & 0.006 & $2.10(1.07-4.11)$ & 0.03 \\
\hline CRRT & $21.05(2.90-152.88)$ & 0.003 & $7.04(1.46-33.85)$ & 0.01 \\
\hline
\end{tabular}

MACCE, major adverse cardiac and cerebrovascular events; MELD score, model for end-stage liver disease score; COPD, chronic obstructive pulmonary disease; NYHA, New York Heart Association; LADd, diastolic dimension of left atrium; CPB, cardiopulmonary bypass; CRRT, continuous renal replacement therapy.

kidneys, and lungs. In the literature, outcomes after ITVS were reported to be mostly derived from single-center case series with variable perioperative mortality ranges of $3-16 \%$ (4-7), one possible explanation for the inconstant outcomes could be the heterogeneous etiologies of TR.

In our series, most patients in the pVHD group underwent cardiac surgery as adults for rheumatic valvular diseases, while most patients in the pCHD group received surgical correction for intracardiac malformation in childhood. Nevertheless, the intervals between the two operations were comparable between the two groups with the median being 16 years, which suggests that the temporary evolution of secondary TR might be independent of initial cardiac pathologies. However, the groups presented with different baseline functional status as revealed by the NYHA classification and ejection fraction 
Table 5 Multivariate Cox regression for all-cause mortality and MACCEs

\begin{tabular}{lcc}
\hline Variables & For all-cause mortality, P value & For MACCEs, P value \\
\hline Interval between two procedures & - & 0.48 \\
MELD score & 0.70 & 0.91 \\
COPD & 0.77 & 0.99 \\
Atrial fibrillation & - & 0.47 \\
EuroSCORE II & 0.79 & - \\
Pre-op NYHA class & 0.64 & 0.12 \\
Pre-op LADd & 0.75 & 0.82 \\
Right ventricular dysfunction & 0.72 & 0.44 \\
CPB time & 0.65 & 0.65 \\
Mechanical ventilation time & 0.92 & - \\
CRRT & 0.84 & 0.97 \\
\hline
\end{tabular}

MACCE, major adverse cardiac and cerebrovascular events; MELD score, model for end-stage liver disease score; COPD, chronic obstructive pulmonary disease; Pre-op, pre-operative; NYHA, New York Heart Association; LADd, left atrial diastolic dimension; CPB, cardiopulmonary bypass; CRRT, continuous renal replacement therapy.

in echocardiography. The onset of atrial fibrillation might be a possible explanation; as we know, atrial fibrillation is per se an important trigger of TR and heart dysfunction. The role of pulmonary hypertension in the natural history of secondary TR following left-sided valvular surgery remains controversial $(1,8)$. The presence of pulmonary hypertension does accelerate the course of secondary TR, but the latter is not necessarily associated with either pulmonary hypertension, or elevated pulmonary resistance as revealed by the available results of right heart catheterization in our study. The precise mechanisms underlying the differential evolution of secondary TR of different etiologies remain to be clarified.

Several techniques aimed at reducing the detrimental effects relative to conventional sternotomy and aortic cross clamping have aroused increasing interest, such as the right anterior thoracotomy approach, beating-heart tricuspid valve surgery, and others. Indeed, long-standing tricuspid insufficiency is frequently associated with liver congestion and more or less coagulopathy; post-operative hemorrhage intrinsically exacerbates right heart dysfunction and compromises the prognosis. Theoretically, right anterior thoracotomy access in the setting of redo surgery is advantageous as compared to median sternotomy since the latter diminishes mediastinal and pericardial dissection, especially when right ventricles adhere to the sternum and right atriums dilate excessively. Previous studies have reported that aortic cross clamping time was an independent predictor of mortality and morbidity in high-risk cardiac patients (9); as a consequence, beating heart tricuspid valve surgery seems to be an attractive strategy to temper the procedural risks; the contemporary vacuum-assisted single venous drainage technique further reduced dissection time. We believe that these minimally invasive techniques possess great potential in the management of isolated tricuspid pathologies, especially in complex cases such as redo surgery.

Deciding how to proceed with tricuspid valve repair or replacement is a major concern in the management of isolated TR. A growing body of evidence suggests that tricuspid valve repair might be associated with lower perioperative mortality as compared to valve replacement (4). We believe that tricuspid valve repair might be the preferred option in concomitant but not in ITVS, especially in the case of redo ITVS following left-sided valvular surgery. The possible difficulty lies in unfavorable anatomy as a result of deformation from left-sided valvular prostheses; in addition, the long-term durability of tricuspid repair is uncertain. Randomized cohort studies are warranted to gain a conclusive insight into this particular issue.

Our study involved several limitations. Due to its retrospective nature, we only included patients who underwent surgical treatment for secondary TR. For one, they mostly represented an entity of "moderate risk" patients since the mean EuroSCORE II calculation was $2.6 \%$; an entity of "high risk" patients refusing or being 
refused for surgery may have been involuntarily excluded; for another, the relatively low peri-operative mortality $(5.2 \%, 3 / 58)$ in our series proved the importance of patients' selection and the timing of surgery. Furthermore, a reliable imaging technique to evaluate right ventricle function was lacking in our study; this assessment is crucial in decisionmaking as patients with advanced right heart failure probably do not benefit from ITVS. However, precise evaluation of right heart function is intrinsically challenging especially in the context of redo surgery, as the presence of left-sided valvular prostheses or tricuspid prostheses increases the difficulty in interpreting the images derived from magnetic resonance imaging or echocardiography. Meanwhile, the baseline characteristics in the primary and secondary TR groups were heterogeneous, but the small sample size impeded our conducting of a propensity score matching study. The relatively short follow-up time might also have increased the probability of type II error. These limitations warrant a larger prospective randomized trial over longer follow-up durations.

\section{Conclusions}

As compared to post-operative TR following surgery for CHDs, TR following left-sided valvular surgery is generally associated with a worse pre-operative functional status and higher surgical risks. However, with the development of surgical techniques and peri-operative management, IVTS can be safely performed in both circumstances with satisfactory mid-term outcomes. Further studies are warranted to explore the mechanisms of differential evolution of secondary TR of different etiologies.

\section{Acknowledgments}

Funding: This work was supported by the National Natural Science Foundation of China (No. 81600229 and 81803059).

\section{Footnote}

Reporting Checklist: The authors have completed the STROBE reporting checklist. Available at http://dx.doi. org/10.21037/jtd-20-1787

Data Sharing Statement: Available at http://dx.doi. org/10.21037/jtd-20-1787
Peer Review File: Available at http://dx.doi.org/10.21037/jtd20-1787

Conflicts of Interest: All authors have completed the ICMJE uniform disclosure form (available at http://dx.doi. org/10.21037/jtd-20-1787). The authors have no conflicts of interest to declare.

Etbical Statement: The authors are accountable for all aspects of the work in ensuring that questions related to the accuracy or integrity of any part of the work are appropriately investigated and resolved. The study was conducted in accordance with the tenets of the Declaration of Helsinki (as revised in 2013), and was approved by the institutional ethics committee (No: IORG0003571). Informed consent was waived because of the retrospective nature of the study and the use of anonymous clinical data for analysis.

Open Access Statement: This is an Open Access article distributed in accordance with the Creative Commons Attribution-NonCommercial-NoDerivs 4.0 International License (CC BY-NC-ND 4.0), which permits the noncommercial replication and distribution of the article with the strict proviso that no changes or edits are made and the original work is properly cited (including links to both the formal publication through the relevant DOI and the license). See: https://creativecommons.org/licenses/by-nc-nd/4.0/.

\section{References}

1. Nath J, Foster E, Heidenreich PA. Impact of tricuspid regurgitation on long-term survival. J Am Coll Cardiol 2004:43:405-9.

2. Antunes MJ, Rodríguez-Palomares J, Prendergast B, et al. Management of tricuspid valve regurgitation. Eur J Cardiothorac Surg 2017;52:1022-30.

3. Rodés-Cabau J, Taramasso M, O'Gara PT. Diagnosis and treatment of tricuspid valve disease: current and future perspectives. Lancet 2016;388:2431-42.

4. Wang TKM, Griffin BP, Miyasaka R, et al. Isolated surgical tricuspid repair versus replacement: meta-analysis of 15069 patients. Open Heart 2020;7:e001227.

5. Raikhelkar J, Lin HM, Neckman D. Isolated Tricuspid Valve Surgery: Predictors of Adverse Outcome and Survival. Heart Lung Circ 2013;22:211-20.

6. Hamandi M, Smith RL, Ryan WH, et al. Outcomes of 
Isolated Tricuspid Valve Surgery Have Improved in the Modern Era. Ann Thorac Surg 2019;108:11-5.

7. De Meester P, Van De Bruaene A, Voigt JU. Outcome and determinants of prognosis in patients undergoing isolated tricuspid valve surgery: Retrospective single center analysis. Int J Cardiol 2014;175:333-9.

8. Lee JW, Song JM, Park JP. Long-Term Prognosis of
Isolated Significant Tricuspid Regurgitation. Circ J 2010;74:375-80.

9. Al-Sarraf N, Thalib L, Hughes A, et al. Cross-clamp time is an independent predictor of mortality and morbidity in lowand high-risk cardiac patients. Int J Surg 2011;9:104-9.

(English Language Editors: J. Jones and J. Gray)
Cite this article as: Cao H, Yang $\mathrm{X}$, Maureira $\mathrm{P}$, Liu J, Liu J, Dan P, Hubert M, Villemot JP, Dong N, Liu Y. Isolated tricuspid valve surgery after congenital versus left heart-disease surgery: mid-term outcomes. J Thorac Dis 2020;12(10):55615570. doi: $10.21037 /$ jtd-20-1787 